\title{
Selective expression of KIR2DL4 for improved diagnosis and as a potential therapeutic target in natural killer/T cell lymphoma
}

\author{
Can Küçük ${ }^{1,2 *}$ and Wing C Chan ${ }^{3}$ \\ ${ }^{1} \dot{I}_{z m i r}$ Biomedicine and Genome (IBG), İzmir, Turkey \\ ${ }^{2}$ Department of Medical Biology, Faculty of Medicine, Dokuz Eylül University, İzmir, Turkey \\ ${ }^{3}$ Department of Pathology, City of Hope Medical Center, Duarte, California, USA
}

Extra-nodal natural killer/T-cell lymphoma, nasal type (NKTCL) is an uncommon but aggressive malignancy associated with significant diagnostic challenges. NKTCLs may occasionally be misdiagnosed as inflammatory diseases that require different disease management and therapeutic strategies. Previous reports indicated NKTCLs can be misdiagnosed as tuberculous meningitis [1], fungal infections [2], or polymyositis [3]. This may result from the frequent small biopsies, massive necrosis, angiocentric tumor infiltrates and unfamiliarity with the condition. Another challenge is related to lack of clonal diagnostic biomarkers for NKCLs, in contrast to B or T-cell malignancies that have uniquely rearranged antigen receptor genes. Therefore, Küçük, et al. recent report [4] that has shown selective expression of KIR2DL4 in more than half of the NKTCL cases often with lack of expression of other KIRs may provide opportunity for more accurate diagnosis.

Killer-cell immunoglobulin like receptors (KIR) belong to a family of polymorphic but homologous genes [5] such that specific measurements of KIR genes, including KIR2DL4 expression are often unreliable using DNA microarray due to non-specific hybridization. Evaluation by antibodies may similarly be compromised due to crossreactivity. Consequently, RNA-Seq applied by Küçük, et al. on NKTCL tumors showed the advantage of this NGS-based method in terms of specificity of measurement of highly homologous gene families such as KIR family genes when coupled with careful analytical approaches. Given the continuously decreasing prices of RNA-Seq in the market, it may be possible in the near future to observe the integration of this NGS-based methodology in routine clinical practice to facilitate NKTCL diagnosis.

Future studies with larger series will need to be evaluated to address whether there is prognostic value of KIR2DL4 expression in addition to its diagnostic utility. Also, it will be interesting to determine the frequency and expression level of KIR2DL4 and other KIRs in extranasal NKTCLs that shows markedly worse prognosis compared to nasal NKTCLs [6].

Another interesting finding presented by Küçük, et al. is the growth inhibition observed after shRNA-mediated knock-down of KIR2DL4 in two malignant NK-cell lines. This observation suggests that KIR2DL4 may transmit activating/survival signals to the neoplastic cells. This

Copyright: (C2018 Küçük C. This is an open-access article distributed under the terms of the Creative Commons Attribution License, which permits unrestricted use, distribution, and reproduction in any medium, provided the original author and source are credited.
KIR is a cell surface receptor normally regulated through engagement with their cognate ligands (e.g. HLA-G) available in the extra-cellular environment. It is not clear whether the regulation of KIR2DL4 only depends on extra-cellular factors or cell-intrinsic mechanisms are present in neoplastic cells. In support of the latter possibility, KIR2DL4 was reported to be present also in endosomes where it can generate signals that can contribute NK-cell activation [7]. More importantly, KIR2DL4 was recently shown to self-associate based on comprehensive characterization of its molecular structure raising the possibility of induction of signal transduction after oligomerization [8]. Altogether these results suggest that KIR2DL4 expression may have biological implications in addition to its potential as a molecular diagnostic marker, and future investigations with higher number of NKTCL patient samples and in-depth functional analysis will be critical to address these possibilities.

\section{References}

1. Cai B, Hu JJ, Tang QX, Lin W, Wang N (2014) Primary meningeal NK/T cell lymphoma masquerading as tuberculous meningitis. Neurol Sci 35: 1467-1469. [Crossref]

2. Zheng Y, Jia J, Li W, Wang J, Tian Q, et al. (2014) Extranodal natural killer/T-cell lymphoma, nasal type, involving the skin, misdiagnosed as nasosinusitis and a funga infection: A case report and literature review. Oncol Lett 8: 2253-2262. [Crossref]

3. Chan EH, Lu SJ, Petersson F, Tan KB, Chng WJ, et al. (2014) Unusual case of metachronous EBV-associated B-cell and NK/T-cell lymphoma mimicking polymyositis-diagnostic challenges and pitfalls. Am J Hematol 89: 110-113. [Crossref]

4. Kucuk C, Hu X, Gong Q, Jiang B, Cornish A, et al. (2016) Diagnostic and Biological Significance of KIR Expression Profile Determined by RNA-Seq in Natural Killer/TCell Lymphoma. Am J Pathol 186: 1435-1441. [Crossref]

5. Yawata M, Yawata N, Abi-Rached L, Parham P (2002) Variation within the human killer cell immunoglobulin-like receptor (KIR) gene family. Crit Rev Immunol 22: 463 482. [Crossref]

6. Au WY, Weisenburger DD, Intragumtornchai T, Nakamura S, Kim WS, et al. (2009) Clinical differences between nasal and extranasal natural killer/T-cell lymphoma: a study of 136 cases from the International Peripheral T-Cell Lymphoma Project. Blood 113: 3931-3937. [Crossref]

7. Rajagopalan S (2010) Endosomal signaling and a novel pathway defined by the natural killer receptor KIR2DL4 (CD158d). Traffic 11: 1381-1390. [Crossref]

8. Moradi S, Berry R, Pymm P, Hitchen C, Beckham SA, et al. (2015) The structure of the atypical killer cell immunoglobulin-like receptor, KIR2DL4. J Biol Chem 290: 1046010471. [Crossref]

*Correspondence to: Can Küçük, $\mathrm{PhD}$, İzmir Biomedicine and Genome Center (IBG), Dokuz Eylül University Health Campus, İzmir / Turkey

Received: June 22, 2018; Accepted: June 27, 2018; Published: June 30, 2018 